cancer in family members. Knowing BRCA status is a very important factor to plan the therapeutic strategy in ovarian cancer.

\section{NIRAPARIB AS MAINTENANCE THERAPY IN PLATINUM- SENSITIVE RECURRENT OVARIAN CANCER: A GEICO STUDY WITHIN THE SPANISH EXTENDED ACCESS PROGRAM}

\begin{abstract}
${ }^{1} \mathrm{JF}$ Cueva Bañuelos*, ${ }^{2}$ Palacio, ${ }^{3} \mathrm{C}$ Churruca, ${ }^{4} \mathrm{~A}$ Herrero-lbáñez, ${ }^{5} \mathrm{~B}$ Pardo, ${ }^{6} \mathrm{M}$ Constenla, ${ }^{7} \mathrm{~A}$ Santaballa, ${ }^{8} \mathrm{~L}$ Manso, ${ }^{9} \mathrm{P}$ Estévez, ${ }^{10} \mathrm{C}$ Maximiano, ${ }^{11} \mathrm{M}$ Legerén, ${ }^{12} \mathrm{G}$ Marquina, ${ }^{13} \mathrm{~A}$ De Juan, ${ }^{14} \mathrm{M}$ Quindós, ${ }^{15} \mathrm{~L}$ Sánchez, ${ }^{16} \mathrm{~A}$ Barquin, ${ }^{17}$ I Fernández Pérez, ${ }^{18} \mathrm{C}$ Martín-Lorente, ${ }^{19} \mathrm{~A}$ Juárez, ${ }^{15} \mathrm{~A}$ González-Martín. ${ }^{1}$ Hospital Clínico Universitario de Santiago de Compostela, Medical Oncology, Santiago de Compostela, Spain; ${ }^{2}$ Hospital Universitario Central de Asturias, Medical Oncology, Oviedo, Spain; ${ }^{3}$ Hospital Universitario Donostia, Medical Oncology, San Sebastián, Spain; ${ }^{4}$ Hospital Universitario Miguel Servet, Medical Oncology, Zaragoza, Spain; ${ }^{5}$ Institut Català d' Oncologia (ICO) Duran i Reynals - IDIBELL, Medical Oncology, Barcelona, Spain; ${ }^{6}$ Complexo Hospitalario Universitario de Pontevedra, Medical Oncology, Pontevedra, Spain; ${ }^{7}$ Hospital Universitari i Politècnic la Fe, Medical Oncology, Valencia, Spain; ${ }^{8}$ Hospital Universitario 12 de Octubre, Medical Oncology, Madrid, Spain; ${ }^{9}$ Hospital Universitario Virgen del Rocío, Medical Oncology, Seville, Spain; ${ }^{10} \mathrm{Hospital}$ Universitario Puerta de Hierro, Medical Oncology, Madrid, Spain; ${ }^{11}$ Hospital Universitario Clínico San Cecilio, Medical Oncology, Granada, Spain; ${ }^{12}$ Hospital Clínico San Carlos, Medical Oncology, Madrid, Spain; ${ }^{13}$ Hospital Universitario Marqués de Valdecilla, Medical Oncology, Santander, Spain; ${ }^{14}$ Complejo Hospitalario Universitario A Coruña, Medical Oncology, A Coruña, Spain; ${ }^{15}$ Clinica Universidad de Navarra, Medical Oncology, Madrid, Spain; ${ }^{16}$ Hospital Universitario HM Sanchinarro, Medical Oncology, Madrid, Spain; ${ }^{17}$ Hospital Universitario Alvaro Cunqueiro, Medical Oncology, Vigo, Spain; ${ }^{18} \mathrm{Hospital}$ de la Santa Creu i Sant Pau, Medical Oncology, Barcelona, Spain; ${ }^{19}$ Hospital Universitari Sant Joan d'Alacant, Medical Oncology, Alicante, Spain
\end{abstract}

\subsection{6/ijgc-2021-ESGO.447}

Introduction/Background* In the ENGOT-OV16/NOVA trial, niraparib demonstrated a significantly longer PFS in patients (pts) with recurrent platinum-sensitive ovarian cancer (PSOC) vs placebo as maintenance therapy, regardless of gBRCA/HRD status. Niraparib obtained EU approval in 2017 and an expanded access program (EAP) was initiated.

Methodology A retrospective study of niraparib maintenance therapy was conducted within the Spanish EAP, at 57 hospitals. Niraparib's safety, dose adjustments, and effectiveness in real-world setting were assessed. Patient characteristics and starting dose individualizations were also analyzed. EAP's inclusion required at least 2 previous courses of platinum-containing therapy. For the last course prior to inclusion a response should have been obtained. Although BRCAmut and BRCAwt pts could be included, most were BRCAwt because olaparib was commercialized at that time for pts with BRCAmut and pts were allowed to be included only in specific circumstances.

Result(s)* Between September 2020 and March 2021, 316 pts were included. Median age was 63 years (31-88). More common initial FIGO stages were IIIC, IVB, and IVA $(50.0 \%$, 13.6\%, 11.1\%). 5.7\% were BRCAmut, 80.4\% BRCAwt, and 13.9\% unknown. 93.4\% had initial surgery and $22.8 \%$ after relapse. Previous bevacizumab was given in $40.8 \%$ of pts. Before niraparib, pts had ECOG 0-1 (50.3\%-47.5\%) and $55.7 \%$ had measurable disease. Population weights $(\mathrm{Kg})$ were 43-57 (23.1\%), 58-76 (52.2\%), and 77-105 (17.7\%). 19.7\% had baseline platelets $<150,000 / \mu \mathrm{L}$, with a median of $203,500 / \mu \mathrm{L}$. Individualized starting dose (ISD) was applied in 203 pts (64.2\%); 142 (70\%) of them started at $200 \mathrm{mg}$. Niraparib's mean dose was $201.5 \mathrm{mg}(59.2 \%$ had $\geq 1$ reduction and $63.3 \% \geq 1$ interruption). $6.0 \%$ discontinued due to niraparib-related adverse events. Main G3-4 hematological toxicities were thrombocytopenia $(17.4 \%$ ISD-32.0\% fixed starting dose (FSD)), anemia (12.4\% $\quad$ ISD-17.5\% $\quad$ FSD), and neutropenia $(7.5 \%$ ISD-5.8\% FSD). There were not relevant G3-4 non-hematological events. 58 (18.3\%) pts were longterm responders (treatment $\geq 1$ year). 47 (14.9\%) remained on treatment upon analysis.

Conclusion* The use of niraparib as maintenance therapy in pts with recurrent PSOC in real-life setting is safe. The ISD approach improved the safety profile. Results were in accordance with those reported in phase III trials. Overall effectiveness analysis is coming.

\section{NON-CODING RNA AND MRNA TRANSCRIPTOME DIFFERENCES IN OVARIAN CARCINOMA PATIENTS ASSOCIATED WITH RESISTANCE TO ADJUVANT CHEMOTHERAPY}

\begin{abstract}
1,2K Seborova* ${ }^{*},{ }^{2} \mathrm{~V}$ Hlavac, ${ }^{3} \mathrm{~L}$ Rob, ${ }^{3} \mathrm{M}$ Hruda, ${ }^{4} \mathrm{~J}$ Bouda, ${ }^{4} \mathrm{P}$ Cernaj, ${ }^{5} \mathrm{M}$ Mrhalova, ${ }^{6}$ I Sedlakova, ${ }^{6}$ S Spacek, ${ }^{1,2}$ P Soucek, ${ }^{1,2} \mathrm{R}$ Vaclavikova. ${ }^{1}$ National Institute of Public Health, Toxicogenomics Unit, Prague, Czech Republic; ${ }^{2}$ Biomedical Center, Faculty of Medicine in Pilsen, Charles University, Laboratory of Pharmacogenomics, Pilsen, Czech Republic; ${ }^{3}$ University Hospital Kralovske Vinohrady, Department of Gynecology and Obstetrics, Prague, Czech Republic; ${ }^{4}$ University Hospital in Pilsen, Charles University, Department of Gynecology and Obstetrics, Pilsen, Czech Republic; ${ }^{5}$ Second Faculty of Medicine, Charles University, Department of Pathology and Molecular Medicine, Prague, Czech Republic; ${ }^{6}$ University Hospital Hradec Králové, Hradec Kralove, Czech Republic
\end{abstract}

\subsection{6/ijgc-2021-ESGO.448}

Introduction/Background* Epithelial ovarian carcinoma (EOC) is associated with the highest mortality among gynecological carcinomas. High mortality is due to the diagnosis at advanced stages and development of resistance to anticancer therapy regimens based on taxanes and platinum derivatives. In effort to overcome the problem of resistance, novel therapeutic drugs are synthetized and new potential therapeutic targets are under study.

Resistance of cancer cells is a multifactorial process, where deregulation of transcriptome may play important role. In addition, long non-coding RNAs (lncRNAs) with recognized regulatory functions may modulate transcriptome profile and its association with resistance and therapy response. The aim of this study was to analyze transcriptome profile of EOC patients and decipher interactions between protein-coding genes and lncRNAs, which may help to reveal new potential therapeutic targets of EOC.

Methodology Set of 60 EOC patients with different sensitivity to the adjuvant chemotherapy was divided into two groups based on their platinum-free interval (PFI) after adjuvant chemotherapy by platinum derivatives in combination with paclitaxel. Together 37 patients had PFI > 12 months (sensitive; $27 \pm 24$ months) and 23 patients had PFI $<12$ months (resistant; $5 \pm 3$ months). We have performed transcriptome profiling using 3'mRNA QuantSeq FWD kit (Lexogen) with sequencing on the NextSeq500 platform (Illumina). Bioinformatics analysis was carried out by in-house pipeline with final differential expression analysis.

Result(s)* Bioinformatics analysis of transcriptome profile revealed significant differences in the expression profile of EOC patients with different sensitivity to adjuvant chemotherapy. We observed twelve differentially expressed protein coding genes (after false discovery rate correction) - MYH11, JAK2, SETDB2, SUCNR1, IRAG2, CCN5, FOXP2, GCNT3, 